\title{
BMJ Open Changes in arterial stiffness indices during a single haemodialysis session in end-stage renal disease population: a systematic review and meta- analysis protocol
}

Catherine Fortier (10 , ${ }^{1,2}$ Hasan Obeid, ${ }^{2}$ Mathilde Paré, ${ }^{2}$ Charles-Antoine Garneau, ${ }^{2}$ Aboubacar Sidibé, ${ }^{2}$ Pierre Boutouyrie, ${ }^{1,3}$ Mohsen Agharazii ${ }^{2,4}$

To cite: Fortier C, Obeid $\mathrm{H}$ Paré $\mathrm{M}$, et al. Changes in arterial stiffness indices during a single haemodialysis session in end-stage renal disease population: a systematic review and metaanalysis protocol. BMJ Open 2021;11:e045912. doi:10.1136/ bmjopen-2020-045912

- Prepublication history and additional supplemental material for this paper are available online. To view these files, please visit the journal online (http://dx.doi.org/10.1136/ bmjopen-2020-045912)

Received 20 0ctober 2020 Accepted 28 July 2021

D) Check for updates

(c) Author(s) (or their employer(s)) 2021. Re-use permitted under CC BY-NC. No commercial re-use. See rights and permissions. Published by BMJ.

For numbered affiliations see end of article.

Correspondence to Dr Mohsen Agharazii; Mohsen.Agharazii@ crchudequebec.ulaval.ca

\section{ABSTRACT}

Introduction Patients with end-stage renal disease are at higher risk of cardiovascular morbidity and mortality, a risk mediated in part by increased aortic stiffness. Arterial stiffness is assessed at different anatomical locations (central elastic or peripheral muscular arteries) using a variety of mechanical biomarkers. However, little is known on the robustness of each of these mechanical biomarkers following a haemodynamic stress caused by a single haemodialysis (HD) session.

Methods and analysis A systematic review has been designed and reported in accordance with the Preferred Reporting Items for Systematic Review and Meta-Analysis Protocols. A targeted search strategy applicable in key databases (PubMed, Embase, the Cochrane Library, Web of Science and grey literature) is constructed to search articles and reviews from inception to 16 0ctober 2020. Only articles of studies conducted with adults under chronic HD for kidney failure, with repeated measures of arterial stiffness metrics (pulse wave velocity, Augmentation Index, arterial distensibility or stiffness) following a before-and-after design surrounding a HD session will be selected. The screening process, data extraction and assessment of risk bias will be done by two independent pairs of reviewers. Meta-analysis will enable adjustments for potential confounders and subgroup analyses will be performed to discriminate changes in arterial stiffness metrics from elastic, muscular or global arterial territories.

Ethics and dissemination This study does not require ethical approval. Findings will be submitted for publication to relevant peer-reviewed journals and will be presented at profession-specific conferences.

PROSPERO registration number CRD42020213946.

\section{INTRODUCTION}

Haemodialysis (HD) is the most common treatment for patients with end-stage renal disease (ESRD). Its intermittent regimen, usually three times per week, leads to inexorable retention of solutes, toxins and excess volume during the interdialytic period
Strengths and limitations of this study

- Selection of before-and-after design studies will enable a better comprehension of the effect of haemodynamic stress that occurs during haemodialysis (HD) session on arterial mechanical properties.

- Subgroup analysis according to site of blood vessels (central elastic vs peripheral muscular) is a relevant approach to explain discrepancies of arterial stiffness changes during HD, as large elastic and medium-sized muscular arteries may behave differently during excess liquid removal and sympathetic activation.

- Meta-regression will help assess the extent of the impact of potential clinical and haemodynamic confounders on the different arterial stiffness indices during a HD session.

- Implementing well-validated scales for the assessment of risk of bias and certainty of evidence will minimise misinterpretation.

- Potential diversity and heterogeneity of arterial stiffness markers may limit quantitative analyses.

(2-3 days), which are partially corrected during the subsequent HD (i.e., usually 4 hours). Despite its vital role, HD is not a physiological treatment. A high ultrafiltration rate during this short period reduces intravascular blood volume leading to a decrease in blood pressure and coronary flow, hypoperfusion of vital vascular beds and reflex activation of sympathetic nervous system which causes tachycardia. ${ }^{1}$ Moreover, during HD, the dialysis membrane is a site where blood has substantial contact with non-biological material, activating white blood cells and their downstream biological reactions which involve activation of complement alternative pathway. ${ }^{23}$ In addition, electrolyte composition of dialysis 
solution may alter cardiovascular response through the acute changes in serum calcium and magnesium concentrations. ${ }^{45}$

Patients with chronic kidney disease are at increased risk of aortic stiffness through various biological processes. ${ }^{6}$ Aortic stiffness is a non-traditional mechanical biomarker of cardiovascular morbidity and mortality, ${ }^{7-9}$ which increases cardiac workload and pulse pressure transmission along the arterial tree. Classically, aortic stiffness is evaluated non-invasively by measuring or estimating carotid-femoral pulse wave velocity (PWV). Other methods aim to quantify the haemodynamic consequences of aortic stiffness through analysis of aortic pulse pressure waveform morphology and determination of Central Augmentation Index as a measure of pressure wave reflection. ${ }^{1011}$ There are also other systems that use heart-ankle PWV or brachial-ankle PWV which incorporates not only the stiffness of aorta (central elastic vessel) but also the stiffness of medium-sized muscular vessels. ${ }^{12}{ }^{13}$ It is also possible to study local arterial stiffness, ${ }^{14}$ for example, by studying pressure-diameter relationship throughout the cardiac cycle for arteries such as the common carotid artery (elastic) or radial artery (muscular). Due to the heterogeneity of the arterial wall composition and dimension, various vascular segments behave differently in response to pathological conditions, volume status, blood pressure, heart rate and sympathetic nervous activity. To what extent a single session of HD affects these measurements is important not only scientifically but also clinically. Inconsistent methodologies and consequent findings not only obscure our understanding of the determinants of vascular stiffness in ESRD, but may also hinder the predictive value of these mechanical biomarkers when assessing cardiovascular risk in this population. ${ }^{15} 16$ Finally, studies addressing this question are scarce and usually include a small number of subjects, which could hamper the reliability of their conclusions. Therefore, we propose to conduct a systematic review and a meta-analysis to estimate the impact of a single session of $\mathrm{HD}$ on markers of arterial stiffness in an attempt to recommend the best timing of measurement with respect to HD. If possible, we will examine whether all vascular segments and markers of arterial stiffness point towards the same conclusion. While pursuing these goals, this review will highlight the strengths and weaknesses of the reported studies, and determine if there is a need for further well-designed investigations.

\section{Objectives}

The major objective of this review is to determine the acute effect of a single HD session on mechanical biomarkers of arterial stiffness including carotid-femoral PWV, carotid-radial PWV, brachial-ankle PWV, femoraltibial PWV, aortic pulse wave analysis, central pulse wave analysis (Augmentation Index and central pulse pressure), aortic/carotid/femoral/radial distension metrics, compliance or incremental elastic modulus.
METHODS

\section{Design}

We will conduct this systematic review and meta-analysis in accordance with this predefined protocol which is reported in line with the Preferred Reporting Items for Systematic Reviews and Meta-Analysis Protocols checklist. ${ }^{17} 18$

\section{Population and eligibility criteria}

In this review, we will include all studies conducted among adult patients ( $\geq 18$ years old) with ESRD undergoing chronic HD, either in hospital setting or at home.

\section{Intervention}

In this review, a single HD session will be considered as the main intervention.

\section{Outcomes}

The primary outcome will be the change in arterial stiffness using PWV-based measurements. PWV is the most widely accepted and used method to measure arterial stiffness by determination of pulse transit time between two points over an arterial segment $(\mathrm{m} / \mathrm{s})$. Arterial segments may include central large elastic and peripheral muscular arteries in different proportions such as carotid-femoral PWV, estimated aortic PWV, brachial-ankle PWV, carotidradial PWV and femoral-distal PWV.

Secondary outcomes will be based on biomarkers of arterial stiffness such central pulse pressure, Central Augmentation Index, arterial distensibility, compliance and incremental elastic modulus of aorta, carotid, femoral and radial arteries. We will report absolute values as well as between-group mean differences in their respective units of measurement per biomarker.

\section{Study design}

We will include all observational studies with repeated measures of arterial stiffness or central pressure with a before-and-after design surrounding a HD session. In the case of interventional studies, the values of the reference group (standard care) will be used in the analysis. We will exclude non-human studies, narrative reviews, in vitro or mathematical modelling reports. Duplicate or substudy of previously published investigations will be removed.

\section{Search strategy}

Our search strategy includes bibliographic databases (PubMed, Embase, The Cochrane Library and Web of Science), references lists of eligible studies and review articles, trials registers and grey literature from inception to 16 October 2020. Medical Subject Headings $(\mathrm{MeSH})$ terms will be used to target articles relevant to the research question. Our proposed literature search strategy is outlined in online supplemental appendix 1 . Manual screening of the reference list will be conducted based on predefined criteria listed in table 1 . No language restrictions or publication period will be imposed on the initial searches; however, our final analysis will be limited to articles originally reported in English, French, 
Table 1 List of inclusion and exclusion criteria for study selection

\begin{tabular}{|c|c|c|}
\hline & Inclusion & Exclusion \\
\hline Population & $\begin{array}{l}\text { Patients with } \\
\text { end-stage renal } \\
\text { disease (stage } 5 \\
\text { CKD) undergoing } \\
\text { haemodialysis } \\
\end{array}$ & $\begin{array}{l}\text { Paediatric population } \\
\text { ( }<18 \text { years old) } \\
\text { Incident haemodialysis } \\
\text { patients ( }<1 \text { month) } \\
\text { Critically ill (eg, } \\
\text { intensive care unit) }\end{array}$ \\
\hline Intervention & $\begin{array}{l}\text { Dialysis } \\
\text { centre-based } \\
\text { haemodialysis } \\
\text { Home-based } \\
\text { haemodialysis }\end{array}$ & $\begin{array}{l}\text { Peritoneal dialysis } \\
\text { Non-standard } \\
\text { haemodialysis } \\
\text { settings (electrolyte } \\
\text { concentrations of } \\
\text { dialysate, temperature, } \\
\text { etc) } \\
\text { Unrelated interventions } \\
\text { such as nutritional, } \\
\text { pharmaceutical and } \\
\text { physical exercise } \\
\text { interventions }\end{array}$ \\
\hline Outcome & $\begin{array}{l}\text { Functional indices } \\
\text { of arterial stiffness: } \\
\text { carotid-femoral } \\
\text { PWV, carotid- } \\
\text { radial PWV, } \\
\text { brachial-ankle } \\
\text { PWV, femoral-tibial } \\
\text { PWV, Cardio- } \\
\text { ankle Vascular } \\
\text { Index, pulse } \\
\text { wave analysis } \\
\text { (Augmentation } \\
\text { Index and central } \\
\text { pulse pressure), } \\
\text { Stiffness Index, } \\
\text { compliance and } \\
\text { distensibility }\end{array}$ & $\begin{array}{l}\text { Peripheral pressure } \\
\text { (brachial, finger, toe, } \\
\text { etc) }\end{array}$ \\
\hline Study design & $\begin{array}{l}\text { Repeated } \\
\text { measures } \\
\text { surrounding } \\
\text { a single } \\
\text { haemodialysis } \\
\text { session } \\
\text { Randomised } \\
\text { controlled and } \\
\text { cross-over trials } \\
\text { (if standard care } \\
\text { group) } \\
\text { Non-randomised } \\
\text { prospective } \\
\text { studies (before- } \\
\text { and-after design) } \\
\text { Articles in English, } \\
\text { French, Italian and } \\
\text { Spanish languages }\end{array}$ & $\begin{array}{l}\text { In vitro or mathematical } \\
\text { modelling reports } \\
\text { Case reports } \\
\text { Animal studies } \\
\text { Substudies of } \\
\text { previously reported } \\
\text { trials } \\
\text { Narrative reviews } \\
\text { Duplicates }\end{array}$ \\
\hline
\end{tabular}

CKD, chronic kidney disease; PWV, pulse wave velocity.

Italian and Spanish. Searches will be rerun just before the final analyses and any further identified studies will be retrieved for inclusion. Unpublished studies will not be sought. Duplicate citations will be removed.

\section{Study screening and exclusions}

An iterative process of study selection will be conducted using the inclusion and exclusion criteria detailed in table 1 . The study selection will be done by two pairs of independent reviewers, each pair screening half of the records. In case of a disagreement between individual judgement, a third reviewer will decide. Decisions will be recorded in an Excel spreadsheet. First, citations will be screened by title and abstract. After this first round of selection, materials and methods sections of the selected articles will be screened to confirm the appropriateness of the study design and of the arterial stiffness assessment method relative to the review question. Before data extraction, another round of selection will be performed by both reviewers at the full-text level.

\section{Data extraction}

A data extraction form will be prepared a priori with consensus among the investigators. Extracted data will include (1) study characteristics, design and methods: title, first and last author, journal and year of publication, research team or country where research was based, language of publication, sources of funding, study design, inclusion and exclusion criteria, time point measurements, type of arterial stiffness instrumentation, method used to identify the foot of the pulse wave when applicable, position of subjects during measurements; (2) sample characteristics: age at the time of measurement, sex distribution, HD vintage, comorbidities (diabetes, hypertension, smoking status, prior history of cardiovascular disease), HD session duration, electrolyte concentration of dialysate (calcium, magnesium), dialysis filter, volume overload; (3) outcomes: peridialytic and intradialytic changes in arterial stiffness based on the above-mentioned methods (carotidfemoral PWV, carotid-radial PWV, brachial-ankle PWV, femoral-tibial PWV, aortic and central pulse wave analysis (Augmentation Index and central pulse pressure), Stiffness Index and local vascular distensibility, compliance and incremental elastic modulus, heart rate and arterial pressure. Study investigators will be contacted by email to gather unreported data or additional details. Extraction of data will be done by two independent reviewers, on separate Excel spreadsheet. Disagreements will be resolved by a third reviewer.

\section{Risk assessment of bias}

Internal validity of randomised controlled trials will be assessed using either the Cochrane Collaboration Risk of Bias tool for randomised controlled trials, the Risk Of Bias in Non-randomised Studies -of Interventions tool (ROBINS-I) for non-randomised studies or the National Institutes of Health quality assessment tool for before-after (pre-post) study without control group. Two reviewers will independently evaluate the possibility of bias in seven different domains including confounding factors (heart rate, mean arterial pressure, fluid removal by HD), selection of participants (unstable participants), classification of the intervention (hypotensive eventfree), deviation from the intended intervention, missing data, measurement of outcomes (seated vs supine) and selection of the reported results. Each domain will be 
judged as either low, moderate, serious or critical risk of bias or no information available. An overall assessment of study bias summarising all domains will be tabulated. A third reviewer will settle unresolved disagreements. In addition, information on the source of funding will be collected to assess conflicts of interest.

\section{Data synthesis and analysis plan}

All studies fulfilling the eligibility criteria will be included in quantitative and qualitative synthesis. Study characteristics will be presented as means and SD or median and IQRs for continuous variables and numbers and percentages for categorical variables. For continuous data, an inverse variance method with random effect models will be used to pool the mean difference or standardised mean difference if studies reported different scales for the assessment of the same outcome. Dichotomous variables will be extracted from individual studies and combined using Mantel-Haenszel method with random effects models to pool relative risks. All analyses will be performed with RevMan V.5.3 (computer program, V.5.3 Copenhagen: The Nordic Cochrane Centre, The Cochrane Collaboration, 2014). Pooled effect sizes and their $95 \%$ confidence limits will be reported. If quantitative synthesis is not appropriate, studies will be described individually according to intervention and outcomes reported in a summary table.

Between-study heterogeneity will be characterised with the Cochrane's I ${ }^{2}$ and will be interpreted as low $(0 \%-30 \%)$, moderate $(30 \%-60 \%)$ and considerable $>60 \%)$.

A meta-regression is planned in case of a considerable heterogeneity among studies and if the number of studies is sufficient ( $>10$ by covariate).$^{18}$ Factors such as age of participants, HD vintage, comorbidities (diabetes, heart failure, etc), amount of liquid overload, heart rate and mean arterial pressure will be considered as covariates if adjusted outcomes are not available or stratification has not been performed. These analyses will be performed using R (R Core Team, 2021) and the metafor Package. ${ }^{19}$.

\section{Sensitivity analysis}

Sensitivity analysis according to study design and high risk of study bias will be performed to explore sources of statistical heterogeneity.

\section{Subgroup analysis}

Peripheral arterial segments are constituted of a higher proportion of vascular smooth muscle cells, in contrast with the high elastin and collagen content of the aorta. Due to intravascular volume correction and sympathetic activation at the end of a HD session, we hypothesised that PWV of central large arteries and peripheral muscular arteries will not respond to the same extent despite adjustments for arterial pressure and heart rate. Therefore, we plan to perform subgroup analysis to pool data of PWV with respect to elastic (aorta), muscularmedium-sized arteries (carotid-radial PWV, femoral-pedal PWV) and global PWV, which includes both elastic and muscular vessels (brachial-ankle PWV, carotid-pedal PWV). We will also plan another subgroup analysis by pooling regional PWV or local biomarkers of arterial stiffness depending on whether the information involves elastic versus muscular vessels.

\section{Meta-bias}

We will attempt to avoid reporting bias by using a sensitive and reproducible search strategy, including as many keywords and synonyms as possible. We will also assess the risk of publication bias with funnel plots if at least 10 studies comparing the same group of treatment are included as recommended by the Cochrane handbook. ${ }^{20}$

\section{Quality of evidence}

To assess the certainty of the evidence and strength of recommendations on the effects of a HD session on arterial stiffness, two reviewers will evaluate the quality of evidence for each outcome measure according to the five domains of the Grading of Recommendations Assessment, Development and Evaluation (GRADE) system. ${ }^{21}$

\section{Amendments}

Any protocol amendments will be summarised in the form of a table, where date of amendment, description of changes and rationale will be provided.

\section{Patient and public involvement}

Patients or the public were not involved in the design, or conduct, or reporting or dissemination plans of this research.

\section{ETHICS AND DISSEMINATION}

This study does not require ethical approval. Findings will be submitted for publication to relevant peer-reviewed journals and will be presented at profession-specific conferences.

\section{CONCLUSIONS}

Patients with ESRD are at high risk of cardiovascular morbidity and mortality, a risk that is mediated in part by increased aortic stiffness, a non-traditional cardiovascular risk factor. Various mechanical biomarkers are used to measure or estimate aortic and arterial stiffness. However, little is known about the robustness of each of these parameters under extreme haemodynamic conditions that occur during a HD treatment. Our review will provide a better understanding of the impact of HD on measures of aortic stiffness and provide the necessary evidence to recommend the most adequate timing of vascular assessment in patients with ESRD.

\section{Author affiliations}

${ }^{1}$ Paris Cardiovascular Research Center (PARCC), Team 7, INSERM-U970, Paris, France

${ }^{2}$ Endocrinology and Nephrology research axis, CHU de Québec-Université Laval Research Center, Québec City, Quebec, Canada 
${ }^{3}$ Pharmacology Unit, Hôpital Européen Georges Pompidou, Université de Paris, APHP, Paris, Île-de-France, France

${ }^{4}$ Division of Nephrology, Department of Medicine, Faculty of Medicine, Université Laval, City of Québec, Quebec, Canada

Contributors All authors contributed towards the submitted final manuscript. CF is a kinesiologist specialised in chronic kidney disease and postdoctoral researcher MA is a nephrologist, the lead supervisor and corresponding author. PB is cosupervisor. $\mathrm{CF}, \mathrm{HO}$ and MP drafted the initial manuscript and received guidance on content, methodology and analysis from AS and MA. MP and C-AG are first reviewers, $\mathrm{CF}$ and $\mathrm{HO}$ are second reviewers. All authors have read and agreed the final manuscript.

Funding The authors have not declared a specific grant for this research from any funding agency in the public, commercial or not-for-profit sectors.

Competing interests None declared.

Patient consent for publication Not required.

Provenance and peer review Not commissioned; externally peer reviewed.

Supplemental material This content has been supplied by the author(s). It has not been vetted by BMJ Publishing Group Limited (BMJ) and may not have been peer-reviewed. Any opinions or recommendations discussed are solely those of the author(s) and are not endorsed by BMJ. BMJ disclaims all liability and responsibility arising from any reliance placed on the content. Where the content includes any translated material, BMJ does not warrant the accuracy and reliability of the translations (including but not limited to local regulations, clinical guidelines, terminology, drug names and drug dosages), and is not responsible for any error and/or omissions arising from translation and adaptation or otherwise.

Open access This is an open access article distributed in accordance with the Creative Commons Attribution Non Commercial (CC BY-NC 4.0) license, which permits others to distribute, remix, adapt, build upon this work non-commercially, and license their derivative works on different terms, provided the original work is properly cited, appropriate credit is given, any changes made indicated, and the use is non-commercial. See: http://creativecommons.org/licenses/by-nc/4.0/.

ORCID iD

Catherine Fortier http://orcid.org/0000-0002-6475-5336

\section{REFERENCES}

1 Rubinger D, Backenroth R, Sapoznikov D. Sympathetic nervous system function and dysfunction in chronic hemodialysis patients. Semin Dial 2013;26:333-43.

2 Ronco C, Clark WR. Haemodialysis membranes. Nat Rev Nephrol 2018;14:394-410.

3 Koga $\mathrm{Y}$, Fujieda $\mathrm{H}$, Meguro $\mathrm{H}$, et al. Biocompatibility of Polysulfone hemodialysis membranes and its mechanisms: involvement of fibrinogen and its integrin receptors in activation of platelets and neutrophils. Artif Organs 2018;42:E246-58.

4 Locatelli F, La Milia V, Violo L, et al. Optimizing haemodialysate composition. Clin Kidney J 2015;8:580-9.

5 Mac-Way F, Leboeuf A, Agharazii M. Arterial stiffness and dialysis calcium concentration. Int J Nephrol 2011;2011:1-6.

6 Zanoli L, Lentini P, Briet M, et al. Arterial stiffness in the heart disease of CKD. J Am Soc Nephrol 2019;30:918-28.

7 Laurent S, Boutouyrie P, Asmar R, et al. Aortic stiffness is an independent predictor of all-cause and cardiovascular mortality in hypertensive patients. Hypertension 2001;37:1236-41.

8 Guérin AP, Pannier B, Métivier F, et al. Assessment and significance of arterial stiffness in patients with chronic kidney disease. Curr Opin Nephrol Hypertens 2008;17:635-41.

9 Pannier B, Guérin AP, Marchais SJ, et al. Stiffness of capacitive and conduit arteries: prognostic significance for end-stage renal disease patients. Hypertension 2005;45:592-6.

10 Loutradis C, Schoina M, Dimitroulas T, et al. Comparison of ambulatory central hemodynamics and arterial stiffness in patients with diabetic and non-diabetic CKD. J Clin Hypertens 2020;22:2239-49.

11 Carlsen RK, Peters CD, Khatir DS, et al. Estimated aortic blood pressure based on radial artery tonometry underestimates directly measured aortic blood pressure in patients with advancing chronic kidney disease staging and increasing arterial stiffness. Kidney Int 2016;90:869-77.

12 Lin M, Chan GC, Chan KW, et al. Vascular age is associated with the risk of dialysis or death in chronic kidney disease. Nephrology 2020;25:314-22.

13 Laurent S, Marais L, Boutouyrie P. The noninvasive assessment of vascular aging. Can J Cardiol 2016;32:669-79.

14 Zanoli L, Empana J-P, Perier M-C, et al. Increased carotid stiffness and remodelling at early stages of chronic kidney disease. $J$ Hypertens 2019;37:1176-82.

15 Bonarjee VVS. Arterial stiffness: a prognostic marker in coronary heart disease. available methods and clinical application. Front Cardiovasc Med 2018;5:64.

16 Laurent S, Cockcroft J, Van Bortel L, et al. Expert consensus document on arterial stiffness: methodological issues and clinical applications. Eur Heart J 2006;27:2588-605.

17 Beller EM, Glasziou PP, Altman DG, et al. PRISMA for Abstracts: reporting systematic reviews in Journal and conference Abstracts. PLoS Med 2013;10:e1001419.

18 Liberati A, Altman DG, Tetzlaff J, et al. The PRISMA statement for reporting systematic reviews and meta-analyses of studies that evaluate healthcare interventions: explanation and elaboration. $B M J$ 2009;339:b2700.

19 Viechtbauer W. Conducting Meta-Analyses in $R$ with the metafor Package. J Stat Softw 2010;36:1-48.

20 Egger M, Davey Smith G, Schneider M, et al. Bias in meta-analysis detected by a simple, graphical test. BMJ 1997;315:629-34.

21 Guyatt GH, Oxman AD, Vist GE, et al. Grade: an emerging consensus on rating quality of evidence and strength of recommendations. $B M J$ 2008;336:924-6. 